\section{In memoriam: Dr. John Last}

\section{En souvenir de $D^{r}$ John Last}

\author{
Louise Potvin $^{1}$ \\ Published online: 3 December 2019 \\ (C) The Canadian Public Health Association 2019
}

"If I have seen further, it is by standing upon the shoulders of giants."

- Sir Isaac Newton

It is with both sadness and a profound sense of gratitude for his outstanding contribution to public health in Canada and worldwide that we mark the passing of Dr. John M. Last. He was professor emeritus at the School of Epidemiology and Public Health, University of Ottawa. The Canadian public health community will remember him as a former Scientific Editor of this journal (1981-1991), a supporter of the Canadian Public Health Association (CPHA), a mentor and tutor to hundreds of students, and a prolific writer and editor whose work will continue to have a lasting impact on public health in Canada and around the world.

For many public health practitioners, the Dictionary of Epidemiology, Public Health and Preventive Medicine, and the Dictionary of Public Health are all well-used tomes that have guided their professional development over the years. Dr. Last's metaphor of the iceberg of disease, first published in The Lancet (Last 1963), is an enduring concept in epidemiological teaching of disease, distinguishing between what is visible or known (clinical epidemiology) and what lurks below the surface (population-based epidemiology). I still use his definition of public health (Last 1998) in my introductory courses, as I have never found a better nor more inspiring one:

Public health is the combination of science, practical skills, and values directed to the maintenance and improvement

Louise Potvin

louise.potvin@umontreal.ca

1 École de santé publique, Université de Montréal et Centre de recherche en santé publique, Montréal, QC, Canada of the health of all the people. It is a set of efforts organized by the society to protect, promote, and restore the people's health through collective or social actions. (...) Public health activities change with changing technology and values, but the goals remain the same - to reduce the amount of disease, premature death and diseaseproduced discomfort and disability in the population.

Beyond the science of public health, his unwavering call was for public health professionals to focus their skills and beliefs on the improvement of the health of all the people. In an interview with Ross Graham (2014) published in this journal, Dr. Last succinctly and eloquently defined the task set before all who choose to practice public health:

...our duty as public health officials is to assemble, assess and evaluate the evidence on public health problems affecting the population we serve. We must show leadership in presenting this evidence to elected officials to whom we are answerable, and must be prepared also to communicate this evidence to the general public, along with actions needed to control the public health problems that we identify. We must be prepared to defend our decisions, which are sometimes opposed by eloquent representatives of powerful vested interest groups. To be effective in this role, we need political savvy - sometimes the most important of the skills we possess.

Never an armchair quarterback, Dr. Last was an ardent defender of the natural environment. His very first editorial in the CJPH as its Scientific Editor started with the words "A safe environment is a prerequisite for good health." (Last 1981). This editorial sparked a conversation that continues today. In the early 1990 s, Dr. Last was a special advisor to the CPHA Task Force on the Implications for Human Health 
of Global Ecological Change (1992). In its final report, the Task Force wrote "Only by becoming part of the growing global movement to protect and enhance human and ecosystem health can we hope to ensure that generations to come will also be able to see the Earth as we see it - a beautiful, living planet - and to be moved by that sight."

As an Editorial Board, we have often taken up Dr. Last's call for advocacy. At a time when public health systems in some provinces and territories are being reorganized and in some cases reduced in size, the resulting changes have raised concern that these systems will no longer be able to meet the current mandate nor address future demands. We have spoken out against these cuts and about why public health remains important. More recently, we have launched a call for papers on the theme "Moving on IPCC $1.5^{\circ} \mathrm{C}$ : Exploring promising public health research, policy, and practice responses to environmental crisis in a warming world", with intended publication as a CJPH Special Section in 2020.

For all of his efforts, Dr. Last received numerous honours and awards - including appointment as an officer in the Order of Canada, the Canadian Public Health Association's R.D. Defries Award, and the Sedgwick gold medal (the American Public Health Association's highest award) - and honorary doctorates from the University of Uppsala in Sweden and the University of Edinburgh in Scotland.

Dr. Last's gift to the world, however, was his passion. It was a passion that he shared with all and a passion that inspired many to do better, to be better.

Louise Potvin

Editor-in-Chief

On behalf of the CJPH Editorial Board

\section{Éditorial}

« Si j'ai vu plus loin, c'est en montant sur les épaules de géants. »

- Sir Isaac Newton

C'est à la fois avec tristesse et une profonde gratitude pour sa contribution exceptionnelle à la santé publique au Canada et dans le monde que nous soulignons le décès de $\mathrm{D}^{\mathrm{r}}$ John M. Last. Il était professeur émérite à l'École d'épidémiologie et de santé publique de l'Université d'Ottawa. La communauté de la santé publique canadienne se souviendra de lui comme d'un ancien rédacteur scientifique de la présente revue (1981-1991), un soutien de l'Association canadienne de santé publique (ACSP), un mentor et un tuteur pour des centaines d'étudiantes et d'étudiants, et un écrivain et directeur de rédaction prolifique dont le travail continuera d'exercer une influence durable sur la santé publique au Canada et à l'étranger.

Pour de nombreux praticiens de la santé publique, son Dictionnaire d'épidémiologie (Dictionary of Epidemiology), son ouvrage Public Health and Preventive Medicine et son Dictionary of Public Health sont des volumes couramment utilisés qui ont guidé leur développement professionnel au fil des ans. Sa métaphore de «l'iceberg de la maladie», parue pour la première fois dans The Lancet (Last 1963), est une notion classique dans l'enseignement de l'épidémiologie des maladies qui établit une distinction entre ce qui est visible ou connu (l'épidémiologie clinique) et ce qui est dissimulé (l'épidémiologie populationnelle). J'utilise encore sa définition de la santé publique (Last 1998) dans mon cours d'introduction, car je n'en ai jamais trouvé de meilleure ni de plus inspirante :

La santé publique est l'un des efforts entrepris par la société pour protéger, promouvoir et rétablir la santé de la population. C'est à la fois un ensemble de sciences, de compétences et de croyances axées sur le maintien et l'amélioration de la santé de toutes les personnes par des actions collectives et sociales. (...) Les activités de santé publique changent au gré des progrès technologiques et de l'évolution des valeurs sociales, mais les objectifs demeurent les mêmes, à savoir réduire la prévalence de la maladie, les décès prématurés de même que l'inconfort et l'incapacité imputables à la maladie dans la population.

Au-delà de la science de la santé publique, son exhortation inconditionnelle aux professionnels de la santé publique était d'appliquer leurs compétences et leurs convictions à l'amélioration de la santé de tous. Dans un entretien avec Ross Graham (2014) paru dans la présente revue, $\mathrm{D}^{\mathrm{r}}$ Last a défini succinctement et avec éloquence la tâche qui attend ceux et celles qui choisissent d'exercer en santé publique :

«...notre devoir de fonctionnaires de la santé publique est de réunir, d'analyser et d'évaluer les données probantes sur les problèmes de santé publique qui touchent la population que nous servons. Il nous faut jouer un rôle d'impulsion en présentant ces données aux élus à qui nous rendons des comptes, et être prêts également à les communiquer au grand public, ainsi que les mesures nécessaires pour maîtriser les problèmes de santé publique que nous avons cernés. Il nous faut être prêts à défendre nos décisions, qui sont souvent combattues par d'éloquents porte-parole de puissants groupes d'intérêts. Pour bien jouer ce rôle, nous avons besoin d'habileté politique - c'est parfois la plus importante corde à notre arc. » [traduction]

Ne jouant jamais les gérants d'estrade, $D^{r}$ Last fut un ardent défendeur des milieux naturels. Son tout premier éditorial 
dans la RCSP à titre de rédacteur scientifique a commencé par ces mots : «Un environnement salubre est une condition essentielle de santé. » (Last 1981). Cet éditorial a suscité un débat qui est toujours d'actualité. Au début des années 1990, $\mathrm{D}^{\mathrm{r}}$ Last a été conseiller spécial du Groupe de travail de l'ACSP sur les répercussions des changements écologiques globaux sur la santé humaine (1992). Dans son rapport final, le groupe écrivait : «Nous devons devenir partie intégrante de ce mouvement global en expansion, qui consacre toutes ses énergies à protéger et à améliorer aussi bien la santé humaine que la santé de l'écosystème. Alors seulement pourrons-nous faire en sorte que les générations à venir puissent voir la terre comme nous la voyons nous-mêmes, une planète vivante et étincelante, et ressentir la même émotion que cette vision nous inspire aujourd'hui. "»

Ici au comité éditorial, nous nous faisons souvent l'écho de l'appel à l'action sociale lancé par $\mathrm{D}^{\mathrm{r}}$ Last. À l'heure où des systèmes de santé publique provinciaux et territoriaux se font réorganiser et parfois réduire, les changements qui en résultent font craindre que ces systèmes ne puissent plus respecter leur mandat actuel ni répondre aux besoins futurs. Nous nous sommes élevés contre ces compressions et nous avons plaidé la cause de l'importance continue de la santé publique. Plus récemment, nous avons lancé un appel d'articles pour une rubrique spéciale de la RCSP à paraître en 2020 : « Réaction au cri d'alarme du GIEC : Des études, des politiques et des pratiques en santé publique prometteuses pour endiguer la crise environnementale posée par une hausse mondiale des températures de $1,5^{\circ} \mathrm{C} »$.

En récompense de ses efforts, $\mathrm{D}^{\mathrm{r}}$ Last a reçu de nombreux prix et distinctions, dont le titre d'officier de
l'Ordre du Canada, le Prix R. D. Defries de l'Association canadienne de santé publique, la médaille d'or Sedgwick (la plus haute distinction de l'American Public Health Association) et des doctorats honoraires de l'Université d'Uppsala en Suède et de l'Université d'Édinbourg en Écosse.

C'est toutefois la passion de $\mathrm{D}^{\mathrm{r}}$ Last qui est son plus grand cadeau à l'humanité, une passion qu'il a partagée avec tous et qui a inspiré beaucoup de gens à faire mieux et à être meilleurs.

Louise Potvin

Rédactrice en chef

Pour le comité éditorial de la RCSP

\section{References/Références bibliographiques}

CPHA Task Force on the Implications for Human Health of Global Ecological Change. (1992). Human \& Ecosystem Health: Canadian Perspectives, Canadian Action. Ottawa: Canadian Public Health Association https:/www.cpha.ca/sites/default/files/ assets/policy/ecosystem_health_e.pdf. Accessed Oct 2019

Graham, R. (2014). Lessons learned and public health's political role: An interview with John Last. Canadian Journal of Public Health, 105(6), 460-461.

Last, J. M. (1963). The iceberg "completing the clinical picture" in general practice. The Lancet, 282(7297), 28-31.

Last, J. M. (1981). Teamwork, leadership and recruitment to public health [Editorial]. Canadian Journal of Public Health, 72, 11-12.

Last, J. M. (1998). Public health and human ecology (Second ed.). Stamford, Connecticut: Appleton \& Lange.

Publisher's note Springer Nature remains neutral with regard to jurisdictional claims in published maps and institutional affiliations. 